\title{
An Argument for Comprehensiveness as the "Special Sauce" in a Recipe for the Patient-Centered Medical Home
}

\author{
Christina Holt, MD, MSc
}

How can we study and quantify potential value that may be added by having primary care specialists (PCSs) from both family medicine and internal medicine provide preventive care services for women? Does seeing one's personal physician (PCP) make a difference? Early answers come from the study by Cohen and Coco in this issue: "Do physicians address other medical problems during preventive gynecologic visits?" Their assessment of a nationally representative sample of preventive gynecological visits uses self-reported data from the physicians to tell us "yes" and to quantify to what extent by specialty, region, and PCP status. This sampling of the content of women's preventive health visits allows us to get a flavor for how the majority of PCSs provide comprehensive care for concomitant acute and chronic issues in the context of individual preventive care visits. Patients are unlikely to parse their presenting concerns into the acute or chronic or preventive categories. The practice of addressing multiple issues during one visit improves the efficiency of addressing patient concerns. This may be a key to the special flavor of a true patient-centered medical home. (J Am Board Fam Med 2014;27:8-10.)

We all recognize clinical sessions similar to these scenarios: a mom comes in for her child's preventive care, but she needs a refill on her contraceptive pill. A teacher is scheduled for a physical examination, has readied himself to quit smoking, but started attending 12-step meetings to cope with anxiety about his wife's excessive drinking and needs to talk. In a visit booked to discuss chronic diabetes, grief and sadness about the patient's elderly mother arise when questions about food choices, time for exercise, and money for test strips are posed. In these cases, we are gratified that the comprehensiveness of our training and the continuity of our relationships allow us to assess and address the patient's concerns and make an appro-

From the Department of Family Medicine, Maine Medical Center, Portland.

Funding: none.

Conflict of interest: none declared.

Corresponding author: Christina Holt, MD, MSc, Research Director, Department of Family Medicine, Maine Medical Center, 272 Congress Street, Portland, ME 04106 (E-mail: holtc@mmc.org).

\section{See Related Article on Page 13.}

priate plan. This often feels like the "special sauce" of family medicine and primary care: there may be many ingredients and potentially effective cooks in the kitchen, but being able to provide for patients' needs at the point of care in a continuous, comprehensive, and contextualized relationship is part of the old recipe of primary care. ${ }^{1}$ Keeping this intact allows the provision of care as a patient-centered medical home $(\mathrm{PCMH})$ to have the sweet taste of home cooking.

If indeed these are critical skills for making an office visit part of a true patient-centered care model, how do we study the frequency or effectiveness of these aspects of our full spectrum of work? Study of administrative billing data can clarify who is attending primary care visits, but does not capture the scope of care provided. When visits cross the boundary of acute issues into preventive care, or preventive care into chronic disease or mental health management, the coding rules become complex and are often not followed. This is potentially due to the extra effort and time required to code for the momentary interactions to address concerns connected to-but essentially distinct from-the main point of the visit. This is 
especially true if the concerns pertain to the patient's relationships with family members or their health needs-the patient's true context of carebut which rarely fit into the main reason for visit. Payers have changing and at times overly complicated arrangements for what can be paid for during any given encounter. Studies show that providers will address more issues than they document and bill for, particularly if they have an ongoing relationship with the patient. ${ }^{2}$

Cohen and $\mathrm{Coco}^{3}$ provide a window into how primary care comprehensiveness may be enacted at specific visits for women's health. Their novel approach analyzed physician self-reported visit content and status as personal physician (PCP) using a nationally representative sample of office visits from the National Center for Health Statistics National Ambulatory Medical Care Survey (NAMCS) and National Hospital Ambulatory Medical Care Survey (NHAMCS). These data provide the opportunity to study the intersection of actual care provided and service utilization, outside of the constraints of specific visit coding choices, which may habitually vary between primary and specialty care. Understanding how care is delivered effectively is essential to PCMH goals of comprehensiveness, continuity, and access, while pointing at the contribution of relationships over time.

Although Cohen and Coco $^{3}$ do not directly study outcomes, a patient's receipt of preventive services in the context of a usual source of care has been shown to be more cost-effective and to prevent the need for hospitalizations, among other higher-cost services. ${ }^{4-6}$ Patient satisfaction also has been linked to having a usual source of care with an ongoing provider relationship. ${ }^{7} \mathrm{We}$ definitely want to know how this benefit develops and measure what we are doing to support these valuable outcomes. The study by Cohen and Coco explicitly quantifies why this may be, with evidence of the frequency with which additional medical concerns are addressed in the preventive care visit by gynecology specialists or PCSs. Their article shows that at least one third of patients who present for cancer screening also seek clarity about other medical problems, and the PCSs address these in the context of the visit more than half the time. PCSs and gynecology specialists are equally likely to address an additional gynecologic concern at a gynecologic preventive visit (12\% of visits), but PCSs are $>3$ times more likely to address $\geq 2$ non-gynecologic con- cerns at these preventive visits. Nearly $90 \%$ of the primary care respondents (family medicine and internal medicine) self-identified as the patient's PCP, and these patients with a usual source of care were more likely to have efficient access to comprehensive care in this sample. These self-reported data from the NAMCS and NHAMCS surveys agree with previous reports that PCSs are more likely to address $>3$ separate issues during visits ${ }^{2}$ and that these cover a broad variety of medical care. Significant shifts in the scope of practice in family medicine have been noted with relation to inpatient and surgical services and maternity and newborn care, but outpatient management across the spectrum of illness remains high, and outpatient visitsregardless of the stated purpose-remain a site for the full spectrum of family-centered care. ${ }^{8,9}$

What can we say about care received outside the context of a PCP or PCMH? While there is some regional variation evident from this study related to proportion of preventive gynecologic visits to PCSs (eg, lower rates in the south, higher rates in rural areas), overall, a much higher proportion of PCSs than gynecologists were the patient's PCP (90\% vs $15 \%)$. Further work should focus on understanding the scope of care provided by those who perceive themselves to be PCPs, and the best way to coordinate the PCMH "neighborhood," or coordination with specialists. ${ }^{10}$ What opportunities for linking acute and chronic care or preventive care might have been lost because of the choice of provider?

Another study of the NAMCS and NHAMCS surveys showed that while ten main diagnoses account for $90 \%$ of visits to urgent care centers, more than $53 \%$ of patients at urgent care have no PCP. When these same diagnoses were the primary reason for care at PCS visits, $19.3 \%$ also included preventive or chronic care provided at the same time. ${ }^{11}$ While access remains a key component for patients at the time services are needed, facilitating comprehensive care for potentially asymptomatic conditions or behaviors is clearly part of the "special sauce" of primary care, leading to less costly and less fragmented comprehensive first-contact care. ${ }^{12}$

Overall, the innovative study of the content and context of preventive care by Cohen and $\mathrm{Coco}^{3}$ adds to our understanding of how variation in the comprehensiveness and complexity of services may differ based on specialty. A "special sauce" for effective primary care in the PCMH has many ingredients: it includes relationships over time, aims to address effi- 
ciently multiple issues, and prioritizes connecting the person with their role within a family or community. While there is no one recipe to mix these seasoned relationships, this article illuminates how one of these ingredients - comprehensiveness - might actually make it into the sauce at the point of care and why we should value the flavor that this adds to the PCMH stew.

\section{References}

1. Loxterkamp D. Benefits of continuity of care. Fam Med 2009;41:312.

2. Beasley JW, Hankey TH, Erickson R, et al. How many problems do family physicians manage at each encounter? A WReN study. Ann Fam Med 2004;2: $405-10$.

3. Cohen D, Coco A. Do physicians address other medical problems during preventive gynecologic visits? J Am Board Fam Med 2014;27:13-8.

4. Saultz JW, Lochner J. Interpersonal continuity of care and care outcomes: a critical review. Ann Fam Med 2005;3:159-66.

5. DeVoe JE, Saultz JW, Krois L, Tillotson CJ. A medical home versus temporary housing: the importance of a stable usual source of care. Pediatrics. 2009;124:1363-71.
6. Clark EC, Saultz J, Buckley DI, Rdesinski R, Goldberg B, Gill JM. The association of family continuity with infant health service use. J Am Board Fam Med 2008;21:385-91.

7. Saultz JW, Albedaiwi W. Interpersonal continuity of care and patient satisfaction: a critical review. Ann Fam Med 2004;2:445-51.

8. Bazemore AW, Petterson S, Johnson N, et al. What services do family physicians provide in a time of primary care transition? J Am Board Fam Med 2011; 24:635-6.

9. Xierali IM, Puffer JC, Tong ST, Bazemore AW, Green LA. The percentage of family physicians attending to women's gender-specific health needs is declining. J Am Board Fam Med 2012;25:406-7.

10. Yee JHF. The patient-centered medical home neighbor: a subspecialty physician's view. Ann Intern Med 2011;154:63-4.

11. Mehrotra A, Wang MC, Lave JR, Adams JL, McGlynn EA. Retail clinics, primary care physicians, and emergency departments: a comparison of patients' visits. Health Aff (Millwood) 2008;27:1272-82.

12. Bennett MD, Applegate WB, Chilton LA, Skipper BJ, White RE. Comparison of family medicine and internal medicine: charges for continuing ambulatory care. Med Care 1983;21:830-9. 